\title{
CHARGE Syndrome Associated with Angle Closure despite High Myopia: A Case Report with Structural Suggestion
}

\author{
Saki Dote ${ }^{a}$ Shunsuke Nakakura ${ }^{a}$ Hirotaka Tanabe $^{a}$ Etsuko Terao ${ }^{a}$ \\ Yuki Nagata $^{a}$ Hitoshi Tabuchi $^{\mathrm{a}}$ Yoshiaki Kiuchi ${ }^{b}$ \\ aDepartment of Ophthalmology, Saneikai Tsukazaki Hospital, Himeji, Japan; \\ bDepartment of Ophthalmology and Visual Science, Graduate School of Biomedical \\ Sciences, Hiroshima University, Hiroshima, Japan
}

\section{Keywords}

CHARGE syndrome $\cdot$ Angle closure glaucoma $\cdot$ Microcornea $\cdot$ High myopia

\begin{abstract}
CHARGE syndrome is associated with multiple malformations, and the main ocular abnormality is coloboma. We describe an unusual case of a 15-year-old girl with CHARGE syndrome having high intraocular pressure and narrow angle closure despite refractive high myopia (-9.0 dpt) in her left eye. Gonioscopy revealed peripheral anterior synechia (approximately $90^{\circ}$ ) in the superior quadrant of the left eye. Both eyes exhibited similar axial length (about $22 \mathrm{~mm}$ ) and corneal curvature (about $8 \mathrm{~mm}$ ). However, microcornea $(9 \mathrm{~mm}$ ), thicker central cornea and iris induced narrower anterior components in the left eye than in the right eye. Preventing the chance of acute primary angle closure attack, the patient underwent laser iridotomy in the left eye; however, long-term follow-up is needed. Additionally, we developed a hypothesis for the mechanism of unilateral angle closure despite high myopia by investigating the ocular structural parameters in detail.




\section{Introduction}

The term CHARGE syndrome was coined on the basis of the multiple malformations common in the disorder: coloboma, heart disease, choanal atresia, retarded growth/development, genital anomalies, and ear abnormalities [1,2]. Approximately $70 \%$ of patients with CHARGE syndrome carry the CHD7 mutation [3,4]. The main ocular abnormalities in CHARGE syndrome are coloboma [5, 6], microphthalmos [5, 6], microcornea [5], ptosis [5, 6], strabismus [6], nystagmus [6], and myopia [5, 6]; regarding glaucoma, only congenital glaucoma has been reported $[7,8]$. Here we report the case of a patient with CHARGE syndrome having unilateral angle closure despite high myopia. By investigating the ocular structural parameters in detail, we were able to develop a hypothesis for the mechanism involved in the development of angle closure.

\section{Case Report}

This study was approved by our local institutional review board (No: 181048) and was performed according to the tenets of the Declaration of Helsinki. Additionally, the patient has provided written informed consent for the publication of the case.

A 15-year-old girl was referred to our hospital with high intraocular pressure and narrow angle closure in her left eye. She had undergone heart surgeries at 2 and 3 months of age. She was also clinically diagnosed with CHARGE syndrome without genetic testing at that time. She exhibited hearing loss, low-set ears, and retarded growth (height, $145.7 \mathrm{~cm}$; weight, $36.0 \mathrm{~kg}$; and $<-2$ standard deviation compared with average 15-year-old Japanese girls), and her mental age was that of a 6-year-old. Her left eye exhibited a smaller palpebral fissure compared with her right eye (Fig. 1, upper panel), and the corneal diameter was 12 and $9 \mathrm{~mm}$ in the right and left eye, respectively. Visual acuity was $0.9\left(1.2 \times \mathrm{S}+2.0 \mathrm{D}=\mathrm{C}-1.0 \mathrm{DA} \times 90^{\circ}\right)$ in the right eye and $0.03\left(0.2 \times \mathrm{S}-8.5 \mathrm{D}=\mathrm{C}-1.0 \mathrm{DA} \times 90^{\circ}\right)$ in the left eye. Using the IcareTA01i rebound tonometer (Tiolat $\mathrm{Oy}$, Helsinki, Finland), intraocular pressure was measured as 12 and $22 \mathrm{~mm}$ $\mathrm{Hg}$ in the right and left eye, respectively. Slit lamp examination revealed normal findings in the right eye. However, shallow anterior chamber (Van Herick, grade 2) and iris depigmentation were noted in the left eye (Fig. 1, second panel). Gonioscopy revealed peripheral anterior synechia (approximately $90^{\circ}$ ) in the superior quadrant of the left eye (Fig. 2, upper right panel); this finding was confirmed using ultrasound biomicroscopy (Fig. 2, bottom right panel). Examination using horizontal scan of swept-source anterior segment optical coherence tomography (SS-1,000 CASIA ${ }^{\mathrm{TM}}$; TOMEY, Nagoya, Japan) revealed that the depth of the anterior chamber was 2.53 and $1.28 \mathrm{~mm}$ in the right and left eye, respectively; further, the central corneal thickness was 0.634 and $0.706 \mathrm{~mm}$, respectively. In addition, the anterior opening distance at $500 \mu \mathrm{m}$ was $0.377 / 0.387 \mathrm{~mm}$ (temporal and nasal) and $0.137 / 0.218 \mathrm{~mm}$, respectively. Iris thickness, measured from the edge of the pupil [9], was as follows: right eye, $0.460 / 0.358 \mathrm{~mm}$ (temporal and nasal) at $1 \mathrm{~mm}$ and $0.410 / 0.287 \mathrm{~mm}$ at $2 \mathrm{~mm}$; left eye, $0.523 / 0.516 \mathrm{~mm}$ at $1 \mathrm{~mm}$ and $0.337 / 0.414 \mathrm{~mm}$ at $2 \mathrm{~mm}$ (Fig. 1, third panel). Axial length, measured using IOLMaster 700 (Carl Zeiss Meditec, Jena, Germany), was 21.90 and $22.27 \mathrm{~mm}$ in the right and left eye, respectively. The average corneal curvature, measured using an autorefractive meter KR-8800 (Topcon, Tokyo, Japan), was 7.88 and $8.01 \mathrm{~mm}$, respectively (Fig. 2, upper left panel). Fundus imaging using Optos 200Tx (Optos PLC, Dunfermline, UK) revealed inferior optic disc coloboma in the right eye and retinochoroidal/optic disc coloboma in the left eye (Fig. 1, fourth panel). B-mode scan using UCD-8000AB (TOMEY, Nagoya, JAPAN) 
was used to detect retinochoroidal coloboma in the left eye (Fig. 1, bottom panel). The associated visual field defect caused by coloboma was confirmed using Goldmann perimetry (Fig. 2, bottom left panel). The patient underwent laser iridotomy at the temporal superior iris in the left eye to prevent any chance of acute primary angle closure attack. Intraocular pressure was slightly decreased to 15-17 $\mathrm{mm} \mathrm{Hg}$ during the follow-up of 6 months after laser iridotomy. The anterior chamber depth and angle parameters were not drastically improved (Fig. 3). Long-term follow-up is needed to assess whether lens removal is required.

\section{Discussion}

This is the first report of a case of angle closure with CHARGE syndrome after our comprehensive and systematic review of the literature using PUBMED. Why did the patient's left eye develop angle closure despite high myopia? Pediatric ophthalmologists may empirically know that microcornea eyes typically have a shallow anterior chamber, thick corneas, and narrow angles and they rarely have angle closure glaucoma. However, the mechanism of this condition is not well elucidated. To answer this question, we investigated the structure of both eyes using the measured parameters shown in Figure 4. Both eyes exhibited almost similar axial length and corneal curvature. The anterior part of the eye was assumed as the same size hemisphere; thus, the corneal transverse diameter affected the degree of angle opening between the eyes. Therefore, microcornea $(9 \mathrm{~mm})$ of the left eye was characterized by a structurally narrower angle than the normal corneal diameter $(12 \mathrm{~mm}$ ) of the right eye (Fig. 4).

Additionally, the thicker central cornea and iris induced narrower anterior components in the left eye versus the right eye. Our assumption was in accordance with previous reports that uveal colobomatous eyes with microcornea have a thicker central cornea than uveal colobomatous eyes with a normal diameter cornea [10]. Additionally, we have previously reported that temporal mean iris thickness values in children (3-16 years) at 1 and $2 \mathrm{~mm}$ were 0.405 and $0.336 \mathrm{~mm}$, respectively. In the nasal angle area, the mean iris thickness values were 0.459 and $0.338 \mathrm{~mm}$, respectively [11], which also supports the thicker iris in the left eye. Therefore, the anterior chamber of the left eye became more cramped compared to the right eye.

The microcornea can also explain the high myopia present in the left eye because the lens is located more anteriorly to the cornea confirmed by anterior segment optical coherence tomography (Fig. 1, third panel), leading to myopia shift compared with the right eye.

A previous study showed that microcornea and high myopia occur in 18.4 and $13.2 \%$ of patients with CHARGE syndrome [5]. The present condition may occur frequently. Therefore, physicians should pay attention to the angle situation when treating patients with CHARGE syndrome, regardless of the refractive error. Also similar to our case, anisometropia ( $>2 \mathrm{dpt}$ ) was found in $89 \%$ ( 8 of 9) and severe mixed myopic astigmatism ( $>3 \mathrm{dpt}$ ), along with large coloboma, was found in 78\% (14 of 18) [12]. Caution should be taken regarding the autorefractive machine that reads variable regions from the coloboma edge to the center of the coloboma cavity, which makes the examination of true refractive error difficult. Microphthalmos (shorter axial length) and microcornea (smaller corneal diameter) are different features; however, some previous reports have shown no apparent difference between them because of no real measurements $[6,13]$. The small appearance of the left eye (Fig. 1, upper panel) does not seem to be caused by ptosis or microphthalmos; it is observed due to microcornea. Sohajda et al. [13] reported a case of angle closure glaucoma with bilateral microcornea ( $8 \mathrm{~mm})$ and high 
myopia in a patient without CHARGE syndrome; however, the hypothesized mechanism of developing angle closure glaucoma was not documented.

Our hypothesis of the angle closure mechanism will be useful for pediatric ophthalmologists who often observe microcornea.

\section{Conclusions}

We showed a rare case of angle closure of a CHARGE syndrome patient and the detailed mechanism of the development of angle closure.

\section{Statement of Ethics}

Written informed consent was obtained from our hospital, Saneikai Tsukazaki Hospital. The authors have no ethical conflicts to disclose.

\section{Disclosure Statement}

The authors have no financial or proprietary interest in any product, method, or material described herein. The authors have no financial disclosures and no conflicts of interest to declare.

\section{Funding Sources}

The authors did not receive any financial support from any public or private sources.

\section{Author Contributions}

Dr. Nakakura had full access to all the data in the study and takes responsibility for the integrity of the data and the accuracy of the data analysis. Study concept and design: Shunsuke Nakakura, Saki Dote, and Yoshiaki Kiuchi. Acquisition, analysis, and interpretation of data: All authors. Drafting of the manuscript: Shunsuke Nakakura. Study supervision: Shunsuke Nakakura, and Yoshiaki Kiuchi.

\section{References}

1 Pagon RA, Graham JM Jr, Zonana J, Yong SL. Coloboma, congenital heart disease, and choanal atresia with multiple anomalies: CHARGE association. J Pediatr. 1981 Aug;99(2):223-7.

2 Pagon RA, Zonana J, Graham JM. CHARGE association. Pediatrics. 1982 Nov;70(5):827-8.

3 Aramaki M, Udaka T, Kosaki R, Makita Y, Okamoto N, Yoshihashi H, et al. Phenotypic spectrum of CHARGE syndrome with CHD7 mutations. J Pediatr. 2006 Mar;148(3):410-4.

4 Lalani SR, Safiullah AM, Fernbach SD, Harutyunyan KG, Thaller C, Peterson LE, et al. Spectrum of CHD7 mutations in 110 individuals with CHARGE syndrome and genotype-phenotype correlation. Am J Hum Genet. 2006 Feb;78(2):303-14.

5 Nishina S, Kosaki R, Yagihashi T, Azuma N, Okamoto N, Hatsukawa Y, et al. Ophthalmic features of CHARGE syndrome with CHD7 mutations. Am J Med Genet A. 2012 Mar;158A(3):514-8. 
6 Russell-Eggitt IM, Blake KD, Taylor DS, Wyse RK. The eye in the CHARGE association. Br J Ophthalmol. 1990 Jul;74(7):421-6.

7 Benson MD, Jivraj I, Damji KF, Solarte CE. Congenital Glaucoma and CHARGE Syndrome: A Case Report. J Glaucoma. 2017 Feb;26(2):e84-6.

8 Katai N, Urakawa Y, Sato Y, Miyanaga K, Segawa K, Yoshimura N. CHARGE association with congenital glaucoma due to maldevelopment of the anterior chamber angle. Acta Ophthalmol Scand. 1997 Jun;75(3):322-4.

9 Nakakura S, Kobayashi Y, Matsuya K, Terao E, Kiuchi Y. Iris thickness and severity of neovascular glaucoma determined using swept-source anterior-segment optical coherence tomography. J Glaucoma. 2018 May;27(5):415-20.

10 Mohamed A, Chaurasia S, Ramappa M, Jalali S. Corneal thickness in uveal coloboma with microcornea. Eye (Lond). 2018 Mar;32(3):586-9.

11 Nakakura S, Nagata Y, Shimizu Y, Kawai A, Tabuchi H, Kiuchi Y. Determination of iris thickness development in children using swept-source anterior-segment optical coherence tomography. PLoS One. 2019 May;14(5):e0217656.

12 McMain K, Blake K, Smith I, Johnson J, Wood E, Tremblay F, et al. Ocular features of CHARGE syndrome. J AAPOS. 2008 Oct;12(5):460-5.

13 Sohajda Z, Holló D, Berta A, Módis L. Microcornea associated with myopia. Graefes Arch Clin Exp Ophthalmol. 2006 Sep;244(9):1211-3. 


\section{Case Reports in Ophthalmology}

\begin{tabular}{l|l}
\hline Case Rep Ophthalmol 2020;11:28-36 \\
\hline DOI: 10.1159/000505389 & $\begin{array}{l}\text { @ 2020 The Author(s). Published by S. Karger AG, Basel } \\
\text { www.karger.com/cop }\end{array}$ \\
\hline
\end{tabular}

Dote et al:: CHARGE Syndrome Associated with Angle Closure despite High Myopia: A Case Report with Structural Suggestion

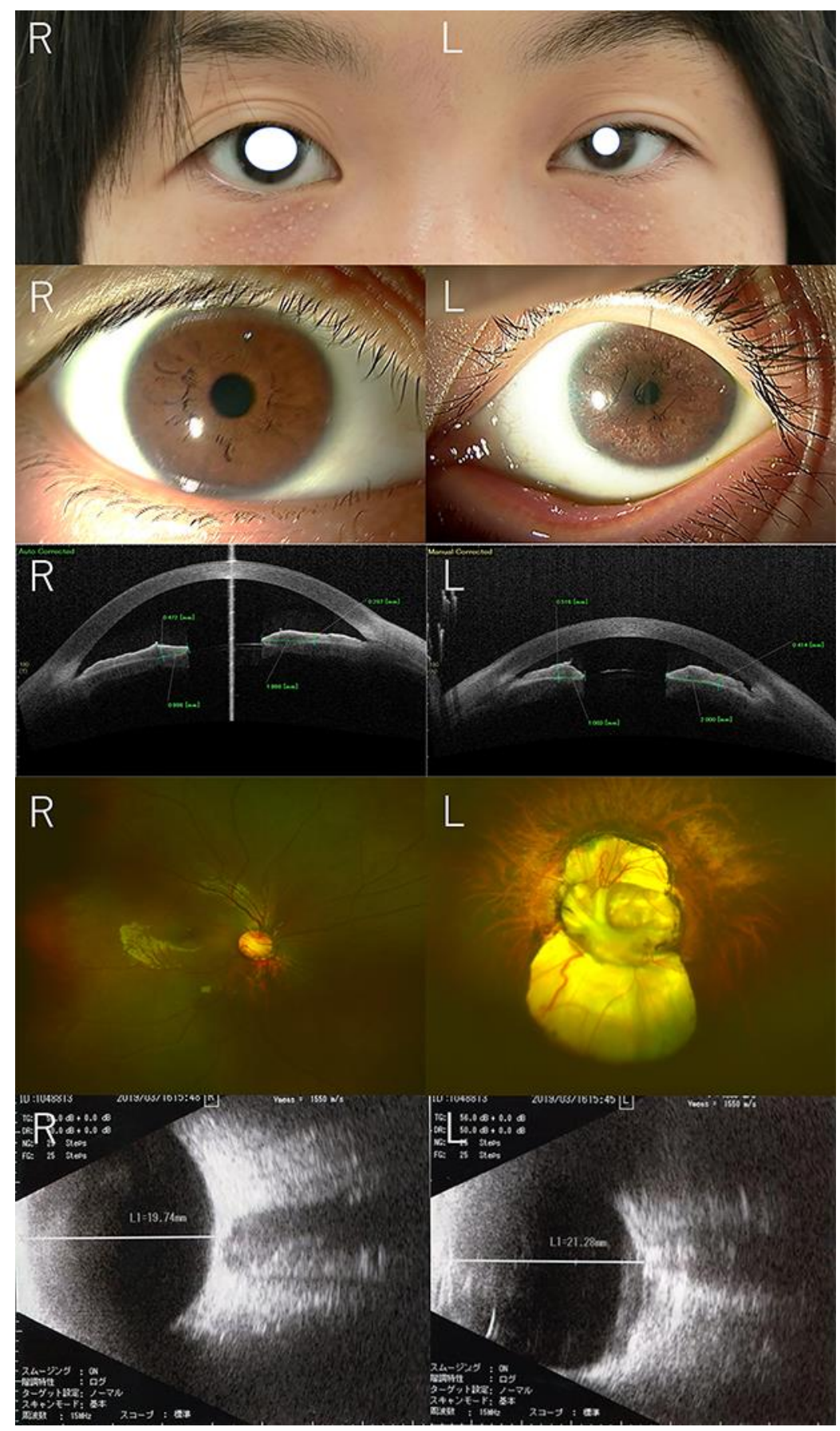

Fig. 1. Ocular findings. Upper panel: facial photograph of the left eye showing microphthalmos due to narrow palpebral fissure or ptosis and apparent microcornea $(9 \mathrm{~mm})$. Second panel: microcornea and depigmentation in the iris of the left eye. Third panel: images of anterior segment optical coherence tomography. Apparent narrow angle, small, shallow anterior chamber and anterior lens positioning were found in the left eye. Fourth panel: disc coloboma in both eyes and retinochoroidal coloboma in the left eye. Bottom panel: B-mode scan roughly showed slightly longer axial length in the left eye $(21.28 \mathrm{~mm})$ than the right eye $(19.74 \mathrm{~mm})$. Note: the axial length was measured from the apex of cornea to disc, not fovea. 


\section{Case Reports in Ophthalmology}

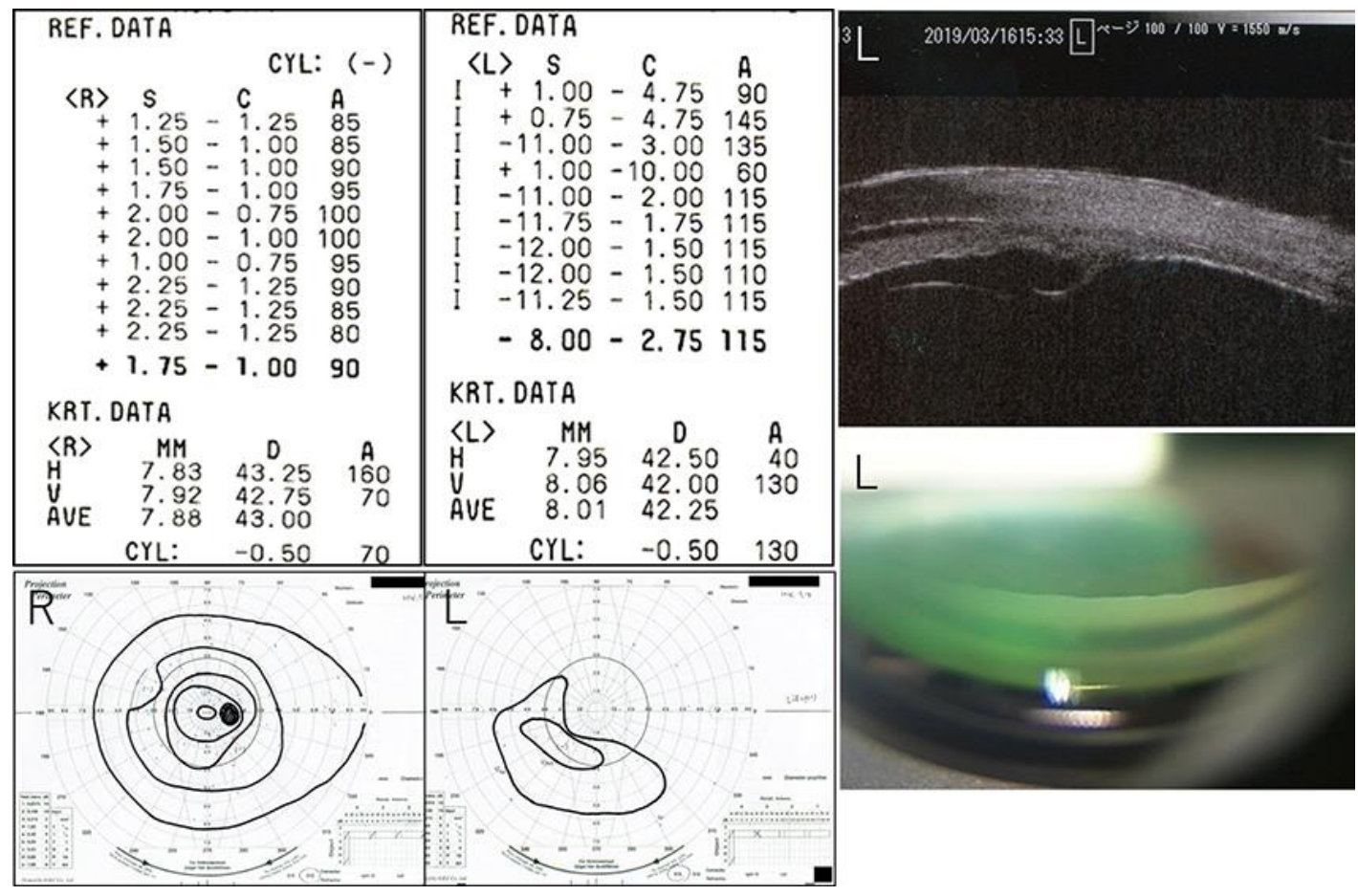

Fig. 2. Refractive error, visual fields, ultrasound biomicroscopy, and gonioscopy. Upper left panel: high myopia in the left eye. Upper right panel: ultrasound biomicroscopy under the dark room condition showing peripheral anterior synechia in the superior angle. Bottom left panel: Goldmann perimetry showing relative scotoma in the right eye and severe visual field defect in the left eye due to coloboma. Bottom right panel: gonioscopy showing peripheral anterior synechia in the superior angle of the left eye. 


\section{Case Reports in Ophthalmology}
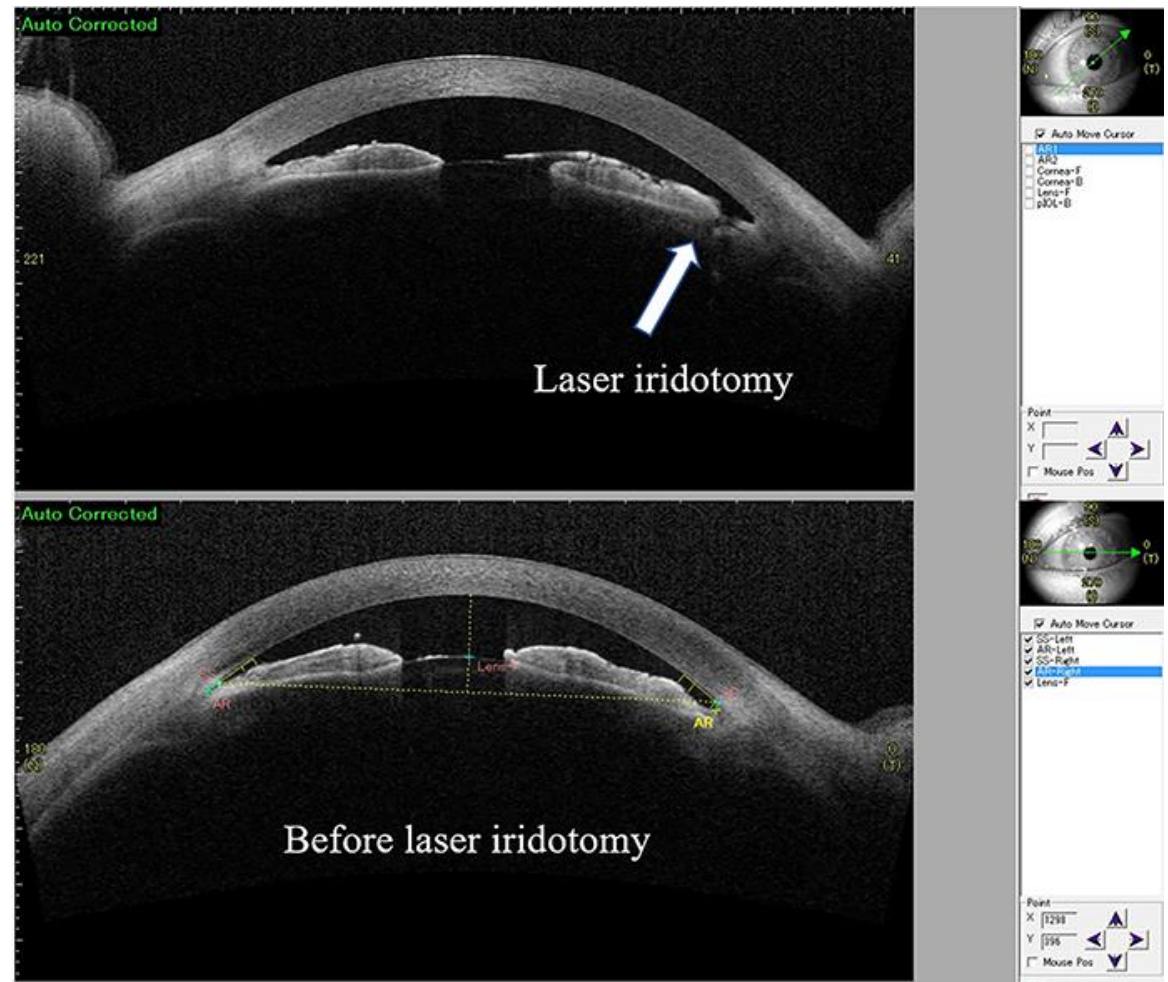

Dote et al: CHARGE Syndrome Associated with Angle Closure despite High Myopia: A Case Report with Structural Suggestion www.karger.com/cop
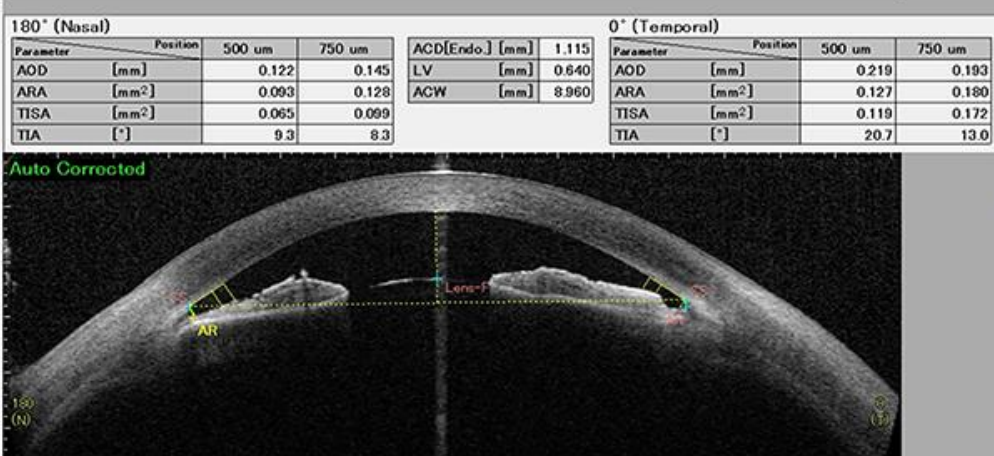

\section{After laser iridotomy}

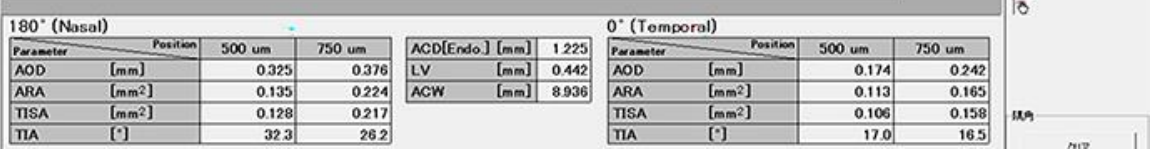

Fig. 3. Anterior chamber changes before/after laser iridotomy in the left eye measured by SS-1,000

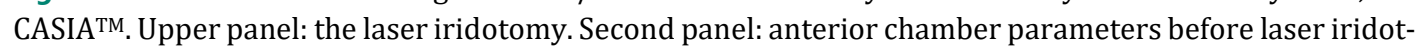
omy. Bottom panel: anterior chamber parameters after laser iridotomy. 
Dote et al.: CHARGE Syndrome Associated with Angle Closure despite High Myopia: A Case Report with Structural Suggestion

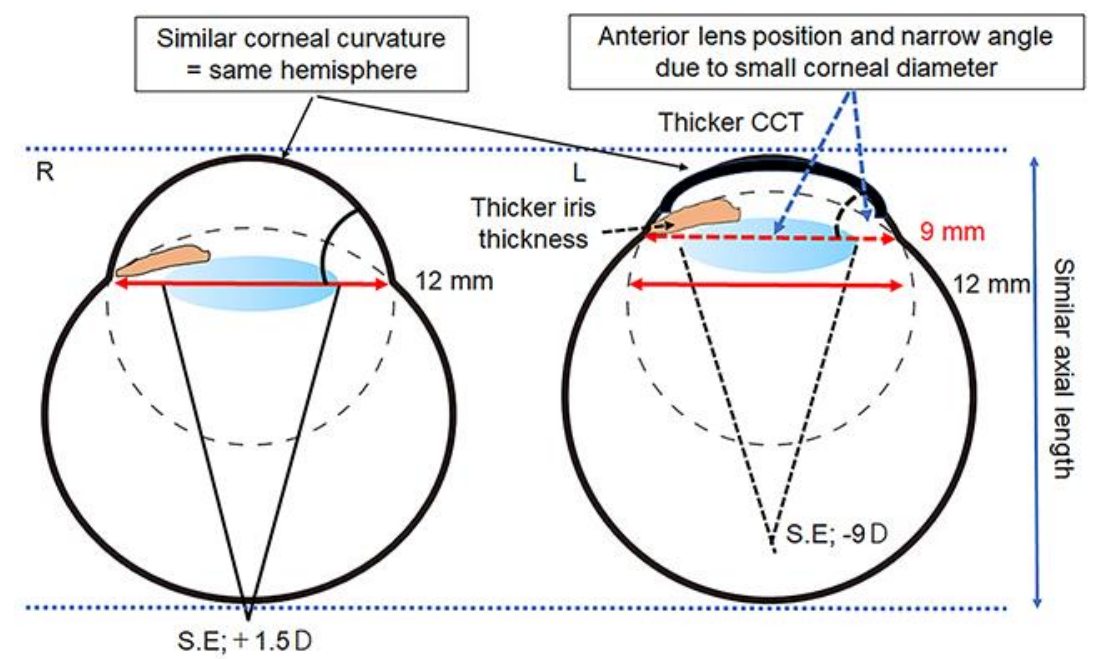

Fig. 4. Schematic drawing of the ocular structure in both eyes. Similar corneal curvature means both eyes have a similar hemisphere. Despite similar axial length, microcornea $(9 \mathrm{~mm})$ induced a narrow angle opening, and thicker central thickness (CCT) and thicker iris thickness add to the small anterior chamber space. The anterior position of the lens in the left eye may explain the high myopia. 